\section{SAT0031 VITAMIN B3 (NAM) SUPPRESSES T CELL ACTIVATION IN AND PRODUCTION OF PRO-INFLAMMATORY CYTOKINES IN VITRO IN A DOSE DEPENDENT MANNER INDICATING THERAPEUTIC POTENTIAL FOR THE TREATMENT OF JIA}

Lotte Nijhuis ${ }^{1}$, Renee van de Wetering ${ }^{1}$, Isabelle Houtzager ${ }^{1}$, Arief Lalmohamed ${ }^{2}$, Sebastian Vastert ${ }^{3}$, Jurg van Loosdregt ${ }^{1} .{ }^{1}$ University Medical Center Utrecht, Laboratory of Translational Immunology, Utrecht, Netherlands; ${ }^{2}$ University Medical Center Utrecht, Clinical pharmacy, Utrecht, Netherlands; ${ }^{3}$ University Medical Center Utrecht, Pediatric Rheumatology and Immunology, Utrecht, Netherlands

Background: Impaired immunological tolerance in Juvenile Idiopathic Arthritis (JIA) is the result of a disturbed balance between regulatory Tcells (Tregs) and effector T-cells (Teffs). Restoring this balance by either enhancing the suppressive function of Tregs or inhibiting activity of proinflammatory Teffs seems a promising therapeutic strategy. We have previously demonstrated that high concentrations of Vitamin B3 (VitB3), also known as nicotinamide (NAM), lead to an increase in FOXP3 positive cells in vitro, via suppression of the deacetylase SIRT1. Because the transcription factor FOXP3 is essential for Treg function, this approach could promote immunological tolerance. We now aim to translate these laboratory findings into clinical practice and envision a role for VitB3 as a novel therapeutic strategy for patients with JIA. However, while VitB3 has promising effects on Tregs, the effect of VitB3 on Teff cells, the other side of the scale, is still unexplored.

Objectives: To investigate the effect of VitB3/SIRT1 inhibition on proliferation, activation, cytokine production and TCR signaling of Teff cells.

Methods: T-cells were isolated from the blood of healthy controls and JIA patients, as well as from the synovial fluid from JIA patients. Cells were stimulated with $\mathrm{aCD} 3 / \mathrm{aCD} 28$ and cultured in the presence of increasing concentrations of VitB3 $(0-9 \mathrm{mM})$ for 1-4 days. Proliferation and expression of activation makers in primary $\mathrm{T}$ cells were determined using flow cytometry. Cytokine production was determined by GPCR, Luminex and flow cytometry. ERK phosphorylation was measured by flow cytometry and Western blot. ERK phosphorylation and GFP expression were also determined by flow cytometry using a murine NFAT-GFP reporter celline.

Results: In vitro VitB3 treatment of JIA patient CD4+ Teff-cells significantly decreased the production of the pro-inflammatory cytokines IL-2 and IFNy measured both on mRNA and protein level. Correspondingly, surface activation markers were downregulated after VitB3 incubation, and ERK phosphorylation was decreased. Furthermore, proliferation of both CD4+ and CD8+ T-cells was inhibited with VitB3 treatment in a dose dependent manner. Murine reporter cells showed similar results. Experimental outcomes were verified using another SIRT1 inhibitor; EX-527.

Conclusion: In addition to the previously demonstrated increase of Treg numbers and functioning, this data demonstrates that VitB3 treatment also inhibits proliferation and activation of Teff cells in vitro. VitB3 treatment could therefore modulate the immunological balance by both increasing tolerance and suppressing immune activation. We envision that VitB3 treatment as an adjuvant therapy has the potential to benefit JIA patients and potentially patients with other autoimmune diseases. To assess the clinical relevance of these findings, we are currently in the preparation of a phase III clinical trial.

Disclosure of Interests: None declared

DOI: 10.1136/annrheumdis-2019-eular.7382

\section{SAT0032 ACTIVATION OF TH LYMPHOCYTES ALTERS THE PATTERN EXPRESSION AND CELLULAR LOCATION OF VIP RECEPTORS IN HEALTHY DONORS AND EARLY ARTHRITIS PATIENTS}

Raúl Villanueva Romero ${ }^{1}$, Irene Gutiérrez-Cañas ${ }^{1}$, Mar Carrión ${ }^{1}$, Isidoro GonzálezÁlvaro $^{2}$, José Miguel Rodríguez Frade ${ }^{3}$, Mario Mellado ${ }^{3}$, María Del Carmen Martínez ${ }^{1}$, Rosa P. Gomariz', Yasmina Juarranz ${ }^{1}$, Cellular and Molecular bases of autoimmune diseases (UCM), Prognostic markers and predictors of therapeutic response in autoimmune diseases (HLP), Chemokine receptors: new targets for therapeutic intervention (CNB). ${ }^{1}$ UCM School of Biological Sciences, Madrid, Spain; ${ }^{2}$ Hospital de La Princesa, Madrid, Spain; ${ }^{3}$ Centro Nacional de Biotecnología, Madrid, Spain

Background: $\mathrm{CD}^{+} \mathrm{T}$ helper (Th) cells are decisive in the struggle against pathogens and in the maintenance of immune homeostasis. Their activation and differentiation can take many forms and generate immune memory in which the extracellular environment plays a critical role in both homeostatic and pathological states. VIP is one of the best-studied neuropeptides with anti-inflammatory and immunomodulatory actions in normal and pathological conditions $(1,2,3)$. There are evidences that VIP may have therapeutic effects for human Rheumatoid arthritis and might be used as a prognostic marker for this disease $(3,4)$. However, there are no deepen studies related to the expression pattern, cellular location, signalling pathways and functionality of its receptors $\left(\mathrm{VPAC}_{1}\right.$ and $\left.\mathrm{VPAC}_{2}\right)$ during the activation of Th cells. Further in-depth studies of their behavio can contribute to the design of new therapies based on their activation and/or blockade.

Objectives: To describe the expression pattern, cellular location and functional role of VIP receptors, VPAC 1 and $\mathrm{VPAC}_{2}$, during the activation of human memory Th cells in healthy conditions and in early arthritis (EA). Methods: Human memory Th cells were isolated from healthy donors (HD) and EA patients. Two experimental conditions, resting state and in vitro activation, were used. Cells were activated (anti-CD3/CD28) and cultured in presence or absence of VIP or specific VPAC receptors agonists. Cellular location of VPAC receptors was examined by western-blot and confocal microscopy, and their mRNA levels were analyzed by semiqPCR. Cytokines, transcription factors and cell markers related to pathogenic profile of Th cells were analyzed by semi-qPCR, ELISA and flow cytometry. To study the signalling pathways, pull-down assays (Rap1), western-blot (CREB) and ELISA (CAMP) were performed. Expression of different chemokines were analyzed by flow cytometry whereas cell migration assays were performed using transwell membranes.

Results: The protein expression pattern of $\mathrm{VPAC}_{1}$ did not change with the activation of Th lymphocytes, whereas $\mathrm{VPAC}_{2}$ was up-regulated. In resting cells, VPAC 1 receptor was located on the plasma membrane and nucleus, while it only appeared in the nucleus in activated cells. VPAC was always found in plasma membrane location. VIP receptors signalled through a PKA-dependent pathway in both conditions, and also through a PKA-independent pathway in activated cells. VIP receptors agonists decreased the pathogenic profile during the activation of Th lymphocytes. Similar results were observed in Th lymphocytes from EA patients.

Conclusion: VIP is an important immunomodulator of $\mathrm{CD4}^{+} \mathrm{T}$ cells in normal and pathological conditions. Our data revealed a change in the expression pattern and cellular location of its receptors (VPAC) during the activation of these cells, giving a wider range of possibilities to dissect its cellular and molecular mechanisms, that would provide new therapies or targets as activity markers in inflammatory/autoinmune diseases.

\section{REFERENCES}

[1] Gomariz RP, et al., Ann NY Acad Sci USA. 2006; 1070: 51-74

[2] Jimeno R, et al. J Leuk Biol. 2015; 98(2): 257-269.

[3] Villanueva-Romero R, et al. J Immunol Res. 2018; 6043710.

[4] Martínez C, et al. Plos One. 2014; 9(1): e85248

Acknowledgement: This work was supported by ISCIII, Spain, co-financed by FEDER (EU): RETICS program, RIER (RD16/0012/0008,0006 and 0011) and the projects (Pl12/00758, Pl14/00477 and Pl17/0027).

Disclosure of Interests: None declared

DOI: 10.1136/annrheumdis-2019-eular.5884

\section{SAT0033 TCR REPERTOIRE PROFILING OF SYNOVIAL FLUID OF HLA-B*27+ AND HLA-B*27- PATIENTS WITH PSORIATIC ARTHRITIS}

Ekaterina Komech ${ }^{1,2}$, Anastasia Koltakova ${ }^{3}$, Alena Novikova ${ }^{1}$, Elena Loginova ${ }^{3}$, Tatiana Korotaeva ${ }^{3}$, Ivan Zvyagin ${ }^{1,2}$. ${ }^{1}$ Shemyakin-Ovchinnikov Institute of Bioorganic Chemistry of RAS, Moscow, Russian Federation; ${ }^{2}$ Pirogov Russian National Research Medical University, Moscow, Russian Federation; ${ }^{3}$ Nasonova Research Institute of Rheumatology, Moscow, Russian Federation

Background: Diagnosis of psoriatic arthritis (PsA) is primarily based on clinical phenotype because of the diversity of the associated features [1] The heterogeneity likely implies different mechanisms of the disease progression or initiation. Certain HLA alleles have been shown as risk factors for different spondyloarthropathies (SpAs), implying role of $\mathrm{T}$ cells in the pathogenesis. The most prominent association have been shown for HLA-B*27 allele with ankylosing spondylitis (AS), giving raise to the arthritogenic peptide theory of the disease initiation. Recently, using deep TCR profiling, new data supporting potential role of CD8+ T cells in AS have been published by our group and others [2,3]. Despite heterogeneous phenotype $24 \%$ of patients from combined cohort of AS and PSA fulfill both CASPAR and modified new-york criteria [4]. Also there is known tendency for axial disease in HLA-B*27+ PsA patients

Objectives: Here we for the first time applied deep TCR profiling to study $T$ cell repertoire of inflamed joints of patients with PsA, to characterise the similarity in clonal composition between patients in presence and absence of HLA-B*27 
Methods: We collected paired $\mathrm{PB}$ and SF samples from $\mathrm{HLA}-\mathrm{B}^{\star} 27+$ $(n=5)$ and HLA-B ${ }^{\star} 27-(n=7)$ patients. All patients fulfilled the CASPAR criteria. TCR beta profiling for total P/SFMC samples and $T$ cell subsets was performed using high throughput sequencing of 5'-RACE double-barcoded TCR beta CDNA libraries following by repertoire reconstruction with molecular barcode-based error correction [5]

Results: Clonal diversity in SF was largely restricted compared to PB with significant enrichment of mostly expanded SF clonotypes compared to $\mathrm{PB}$, suggesting antigen-driven migration and expansion of the $\mathrm{T}$ cells into inflamed site. Major clonal expansions of SF were private for each patient, yet SF shared significantly more clonotypes than $\mathrm{PB}$ independently from $H L A-B^{\star} 27$ status. Most important, in CD8+ subsets from PB and SF we identified the previously described AS-associated T-cell clonotypes. These clonotypes were detected in SF of all HLA-B*27+ patients but not in samples from HLA-B*27- patients, suggesting the HLA-B*27 restriction of the TCR beta motif. The clonotypes were enriched in SF compared to $\mathrm{PB}$, representing up to $1.6 \%$ of $\mathrm{CD} 8+\mathrm{SF} \mathrm{T}$ cells. All HLA$B^{*} 27+$ patients had several variants of the TCR beta clonotypes in SF, suggesting the selection of the TCRs during disease development

Conclusion: Our results further support the supposed role of the CD8+ T cells in SpAs and suggest that similarity of clinical phenotype between AS and HLA-B ${ }^{*} 27+$ PsA patients could be associated with autoimmune response to similar antigens, represented by $\mathrm{HLA}-\mathrm{B}^{\star} 27$ molecule

\section{REFERENCES}

[1] Veale D. J. and Fearon U. (2018) 'The pathogenesis of psoriatic arthritis', Lancet (London, England). Elsevier Ltd, 391(10136), pp.2273-84

[2] Faham M. et al. (2016) 'Discovery of T-Cell Receptor Beta Motifs Specific to HLA-B27 + Ankylosing Spondylitis by Deep Repertoire Sequence Analysis', Arthritis \& Rheumatology, 11(10), pp.300-8

[3] Komech E. A. et al. (2018) 'CD8+ T cells with characteristic T cell receptor beta motif are detected in blood and expanded in synovial fluid of ankylosing spondylitis patients', Rheumatology (Oxford), 57(6), pp.1097-104

[4] Jadon D. R. et al. (2017) 'Axial Disease in Psoriatic Arthritis study: Defining the clinical and radiographic phenotype of psoriatic spondyloarthritis', Annals of the Rheumatic Diseases, 76(4), pp.701-7

[5] Zvyagin I.V. et al. (2017) 'Tracking T-cell immune reconstitution after TCR $\alpha \beta / C D 19-d e p l e t e d$ hematopoietic cells transplantation in children', Leukemia, 31(5), pp.1145-53

Acknowledgement: Funding: RSF grant No 17-75-10220

Disclosure of Interests: Ekaterina Komech: None declared, Anastasia Koltakova: None declared, Alena Novikova: None declared, Elena Loginova Speakers bureau: Novartis, Celgene Corporation, Biocad, Janssen, AbbVie Inc, Tatiana Korotaeva Speakers bureau: Novartis, Celgene Corporation, AbbVie Inc, Biocad, Janssen, Pfizer, UCB, Lilly, Ivan Zvyagin: None declared

DOI: 10.1136/annrheumdis-2019-eular.4212

\section{Rheumatoid arthritis - etiology, pathogenesis and animal models}

\section{SAT0035 \\ IS PHARMACOLOGICAL CLINICAL REMISSION SYNONYMOUS WITH BIOLOGICAL INACTIVE DISEASE? DIFFERENTIAL GENE EXPRESSION ANALYSIS IN RHEUMATOID ARTHRITISAND HEALTY CONTROLS}

Maria Maddalena Angioni, Daniela Perra, Enrico Mascia, Silvia Pinna, Ignazio Cangemi, Piero Mascia, Mattia Congia, Alberto Floris, Matteo Piga, Alessandro Mathieu, Alberto Cauli. Università degli Studi ed AOU di Cagliari, Dip.to Scienze Mediche e Sanita' Pubblica, Unità di Reumatologia, Monserrato (Cagliari), Italy

Background: Remission is an important goal of therapy in rheumatoid arthritis (RA), but data on molecular players of clinical remission and effective disease inactivation are scarce. Gene expression profiling analysis is useful to elucidate the pathogenic mechanisms of diseases, and differential gene expression analysis between diverse disease conditions produces gene signatures characteristic of the state or disease being studied.

Objectives: Our aim was to compare the transcriptional profiles of patients with clinically active versus inactive (remission state) RA, and healthy controls (HCs).

Methods: From a cohort of around 1000 patients affected by RA according to ACR-EULAR 2010 criteria, we first selected 20 patients with active disease state (without biologic treatment ongoing) (A) and 20 patients with $>1$-year remission induced by TNF $\alpha$ antagonism (Etanercept) (R), as assessed by DAS28(PCR) scores, and from $20 \mathrm{HCs}$ matching for age and gender ratio. Both RA groups were not on corticosteroid treatment. RNA from peripheral blood was extracted and, following quality analysis by Agilent Bioanalyzer, each condition has been profiled using RNAs pools in biological duplicates by distinct Affymetrix Human GeneChip HTA 2.0, for a total of 6 arrays. Data analysis was performed using the commercial software Partek Genomics Suite, V 6.6. To identify a transcript as differentially expressed, a value of fold change 1.5 and $p$-value 0.05 has been set.

Results: The Venn diagram shows all comparative groups (A vs R, A vs $\mathrm{HC}, \quad \mathrm{R}$ vs $\mathrm{HC}$ ) with their relative amount of transcripts differentially expressed, generated using abovementioned parameters, and the relationship between sets (fig1, panel A). Using the list of transcripts differentially expressed in at least one of the aforementioned comparison, a hierarchical clustering was carried out to view the intra-condition expression profile. Here, we have identified (arbitrarily) 4 clusters of transcripts with analogous transcriptional profile and to each of them a color code has been assigned (Heatmap in Fig1, panel B). For these clusters and for all lists of transcripts differentially expressed founded by our comparative study, we carried out the Gene Set Enrichment Analysis by Gene Ontology (GO), in order to identify how molecular functions, cellular compo nents or biological processes occurs more frequently than expected in a reference list of transcripts.

Conclusion: Considering the amount of differentially expressed transcripts and the hierarchical clustering analysis, is evident that the drug-induced remission $(R)$ is more similar with $\mathrm{HCs}$ condition, while active disease state (A) has a different profile; however "similar" profile does not mean "identical". In fact, the Gene Set Enrichment Analysis Score showed us that mRNA transcripts dysregulated in the $\mathrm{R}$ condition vs $\mathrm{HCs}$, are involved in several biological processes regarding the immune system, response to stimulus, biological regulation, locomotion and others. Our next step will be to validate, by Real Time PCR in a large cohort of patients, the most interesting dysregulated genes covering biological func tions eventually sustaining disease activity.

\section{A}

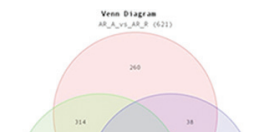

B

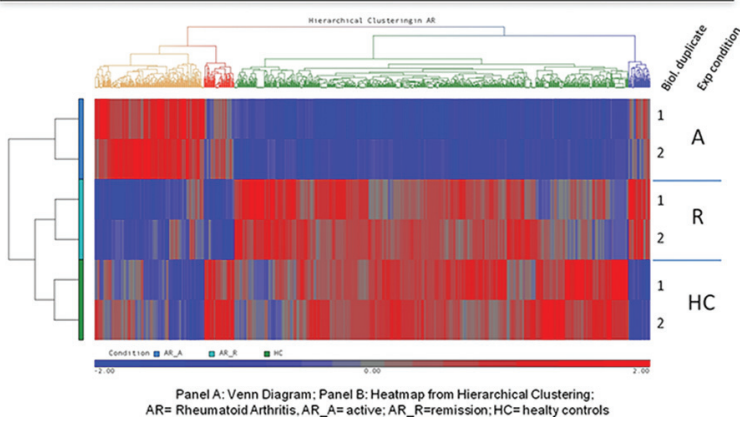

Disclosure of Interests: None declared DOI: 10.1136/annrheumdis-2019-eular.5652 